Cahiers de philosophie de l'université de

CAHIERS DE PHILOSOPHIE
DE LUNIVERSITE DE CAEN

Caen

49 | 2012

Levinas : au-delà du visible

\title{
Penser à l'envers : la genèse du renversement de la philosophie chez Emmanuel Levinas
}

Carla Canullo

\section{(2) OpenEdition}

1 Journals

Édition électronique

URL : https://journals.openedition.org/cpuc/888

DOI : $10.4000 /$ cpuc. 888

ISSN : 2677-6529

Éditeur

Presses universitaires de Caen

Édition imprimée

Date de publication : 31 juillet 2012

Pagination : 255-282

ISBN : 978-2-84133-410-0

ISSN : 1282-6545

Référence électronique

Carla Canullo, «Penser à l'envers : la genèse du renversement de la philosophie chez Emmanuel Levinas ", Cahiers de philosophie de l'université de Caen [En ligne], 49 | 2012, mis en ligne le 07 juin 2018, consulté le 01 février 2023. URL : http://journals.openedition.org/cpuc/888 ; DOI : https://doi.org/ 10.4000/cpuc.888

\section{(c) (†) 8}

Creative Commons - Attribution - Pas d'Utilisation Commerciale 4.0 International - CC BY-NC 4.0 https://creativecommons.org/licenses/by-nc/4.0/ 


\section{Penser à l'envers: la genèse du renversement de la philosophie chez Emmanuel Levinas}

\section{Le renversement en question}

La lecture que nous allons proposer a lieu dans le cadre d'une interprétation du mouvement phénoménologique français, auquel la communauté scientifique internationale, depuis quelques années, a consacré une grande attention; un mouvement qui semble avoir mis en ouvre un renversement de la phénoménologie historique de Husserl et de Heidegger. La possibilité d'une contre-intentionnalité, le déplacement du sujet du nominatif à l'accusatif, l'hypothèse que la subjectivité est constituée et non constituante: voici quelques motifs originaux présentés par la phénoménologie française contemporaine d'après lesquels on a interrogé l'élargissement de la phénoménalité au-delà des horizons déjà pensés pour saisir sa donation. Il reste à vérifier si ce renversement est déjà préfiguré dans l'œuvre d'Emmanuel Levinas, non tant en terme de renversement de la phénoménologie, mais en tant que renversement de la philosophie elle-même, de son attitude grecque, moderne ou phénoménologique. Un renversement que l'on propose de concevoir en deux sens, suivant les mots allemands qui en expriment la signification. En tant que Umkehrung, mot qui indique, tout comme le verbe umkehren, le fait de renverser et bouleverser mais aussi d'invertir. Il s'agit d'un renversement que l'on peut nommer thématique, dans la mesure où il est une inversion de l'ordre des termes du raisonnement pour formuler des questions déjà posées, tout en les renouvelant. Cependant, le renversement peut être conçu aussi comme Umschlag, mot qui fait allusion, comme dans l'enveloppe, à un 
accomplissement qui est un envelopper et protéger, mais aussi un «se renverser» (tout comme l'enveloppe a à se renverser pour accueillir et protéger son contenu). Dans la figure de l'Umschlag, les deux sens du mot allemand se rejoignent, indiquant aussi bien le renversement que l'enveloppe, l'étui. Un renversement qui, certes, est thématique, mais aussi philosophique, par lequel - grâce à l'inversion thématique - on cherche à atteindre un envers jusqu'à ce moment invu ${ }^{1}$, un en-soi que la philosophie, par ses catégories et ses horizons, n’avait pas encore aperçu.

Dès lors, il y a un renversement de la philosophie en tant qu' "avènement» de celle-ci par le questionnement de ses sujets et problèmes et de ses propres questions. Il faut encore comprendre le renversement en tant que "garde» de questions qui, par leur même questionnement et par le fait même de se questionner, demeurent toujours et à nouveau possibles, donc livrées et, en même temps, gardées et protégées. Dans les Inédits d'Emmanuel Levinas, dans la genèse de cette pensée, il sera possible de vérifier ces deux sens du renversement, en cherchant à apercevoir ce qu'il en est du renversement thématique (et du point de vue philosophique différent qu'il inaugure), donc du renversement ou inversion de la méthode philosophique, et ce qu'il en est de l'envers atteint par cette inversion (et donc «vers où " perce ce renversement de la philosophie). Envers en tant qu'en-soi invu et insaisissable autrement que par le renversement - thématique et philosophique dans le sens plus strict du terme - que lui-même provoque. Il ne s'agit donc pas d'un envers qui serait un en-soi atteint et saisi, mais d'un envers qui se donne à atteindre et à saisir par une réversibilité intrinsèque qu'il reste à définir.

Les termes relatifs au renversement thématique reviennent maintes fois et dans des passages différents, dans le premier volume des Inédits aussi bien que dans le deuxième. Le lexique du renversement thématique est le suivant: inversion, bouleversement, retournement (qui revient avec l'expression «retourner la réflexion»), revirement, virement et, bien entendu, renversement et renverser les termes. Annonçant ce que l'on pourrait caractériser comme renversement thématique, Levinas dit explicitement qu' «il faut renverser les termes $»^{2}$. Cela est mentionné en vue de la mise en place d'une pensée de la temporalité en tant que simultanéité et des percées qu'elle donne à penser. «J'en viens à renverser l'ordre ${ }^{3}$, lit-on dans la note $<30>$

1. Voir J.-L. Marion, De surcroît, Paris, PUF, 2001 : «Par "invu", nous entendons purement et simplement ce qui, de fait, ne parvient pas ou pas encore à la visibilité, alors que je pourrais de droit l'expérimenter comme un possible visible».

2. E. Levinas, Notes philosophiques diverses, in Carnets de captivité, suivi de Écrits sur la captivité et Notes philosophiques diverses (= Euvres 1), R. Calin et C. Chalier (éd.), Paris, Grasset - IMEC, 2009, p. 411.

3. Ibid., p. 396 . 
de la liasse $\mathrm{C}$ des Notes philosophiques diverses, où se présentent d'autres thèmes centraux de la pensée d'Emmanuel Levinas, à savoir l'extériorité et l'infini ${ }^{4}$. Dans la conférence "Le Vouloir», les termes du renversement thématique reviennent dans ses multiples formulations (retournement, virement, revirement) indiquant «le mouvement radicalement différent de celui de la pensée», c'est-à-dire le mouvement renversant la primauté de la pensée constituante dans une phénoménologie de l'habitation où le constitué «déborde son sens ${ }^{5}$ :

Je voudrais tenter la phénoménologie de l'habitation par laquelle le sujet est chez soi et se constitue comme personne et comme volonté. Habiter, cela consiste en ceci: ce qui est objet de ma pensée, \{ce qui\} a un sens pour ma pensée et \{ce qui\} se constitue comme ma représentation - le constitué déborde son sens. Le \{sens\} constitué devient au sein de la constitution, la condition et la nourriture du constituant. [...] Il y a ici un retournement unique: comme si une pensée constituante se piquait à son jeu dans son jeu libre de la constitution, comme si la liberté de la pensée, $\{-\}$ commencement total ou présent $\{-\}$ se trouvait une condition dans son propre produit. La situation est radicalement opposée à celle où on prête à un objet un sens d'être ${ }^{6}$.

Autre par rapport à la "phénoménologie husserlienne qui ne voit pas de limites à la noématisation ${ }^{7}$,

[ce] revirement de la représentation en habitation, marque \{un\} rapport avec l'être qu'il est précisément impossible de caractériser par la conscience de et qui, au lieu de se résumer en extase - en transcendance - en passage vers quelque chose d'extérieur est une intériorisation, une rentrée dans... une séparation de l'extérieur, l'habitation ${ }^{8}$.

Plus loin Levinas ajoute:

Le virement n'enseigne rien sur l'objet de la pensée, il n'est pas connaissance - il consiste précisément à invertir l'objectivation, il est une fonction dans la structure du sujet par laquelle le sujet est sujet séparé, est ici, est le même9 ${ }^{9}$

4. «J'en viens à renverser l'ordre. Ce n'est pas ce qui est infini qui est extérieur, mais ce qui est extérieur qui est infini. L'être - c'est se rapporter à l'extérieur. Le rapport avec l'extérieur - le rapport moral - est la condition de la liberté. La communication des individus est la source de la conscience» (ibid.).

5. E. Levinas, "Le Vouloir», in Parole et Silence et autres conférences inédites au Collège philosophique (= Euvres 2), R. Calin et C. Chalier (éd.), Paris, Grasset - IMEC, 2011, p. 240.

6. Ibid.

7. Ibid., p. 241. Il s'agit de passages dans lesquels l'habitation est le thème principal ainsi que "le revirement de la représentation en habitation».

8. Ibid.

9. Ibid 
D'après ce passage, semblerait s'annoncer le caractère formel et purement thématique du "virement», le fait qu'il ne s'attache pas à la connaissance «de quelque chose», mais au "comment» le sujet se caractérise par cette fonction invertissant l'objectivation elle-même, et donc à une inversion s'accomplissant non seulement pour (mieux) connaître, mais encore par le connaître. Il s'agit d'une fonction du sujet le caractérisant en propre, car elle détermine sa structure théorétique.

Cependant, qu'il s'agisse d'une fonction du sujet n'implique pas que celui-ci opère ce renversement, qui est aussi une inversion, par soi et grâce à soi. Au contraire, la perspective dans laquelle la question trouve son origine se dévoile ici, et cette perspective est différente de celle du renversement qui, pourtant, l'annonce: l'orientation. Voilà formulée une question qui, dans les Inédits, se pose a contrario quand - sur le recto d'une carte d'invitation de 1954 - Levinas écrit: «Chez Heidegger - pensée de l'être - pas d'orientation! $\aleph^{10}$ - alors que la question de l'orientation dans la pensée heideggérienne se pose explicitement ${ }^{11}$. Cependant, Levinas, à l'encontre de Heidegger, pose la question de l'orientation ailleurs que sur l'horizon de l'être. Surtout, il la pose explicitement là où il pose la question du sens, qui ne doit pas être compris comme le sens d'être, car surgissant, bien au contraire, «quand de l'être s'obscurcit, d'une désorientation $»^{12}$. À nouveau, dans le douzième point de cette même note $(<8>)$ des Notes philosophiques diverses, Levinas écrit $:$ "Le sens $=$ l'être est orienté. Sens $=$ orientation. Être orienté $=$ agir ${ }^{13}$. Plus loin il ajoute: «La vie est d'emblée orientée» et «Action (= orientation totale) suppose a) pas de retour sur soi (pas de pensée au sens absolu du terme, pouvant absorber l'attitude) b) aboutissement, prise sur le réel (sans quoi l'action serait jeu) $»^{14}$. Pour arriver, enfin, à la proposition d'une liste des significations de l'orientation (points 16 à 26), qui commence avec le sens et aboutit à la signification, le point cardinal de l'orientation n'étant pas (dans) le sujet mais s'identifiant à Autrui: «Terme de l'orientation: absolu = visage présent en personne. Source de sens - Autrui $»^{15}$. Le virement radical se joue ici, dans ce choix ou dans cette option du philosophe le menant du terme de l'orientation vers le «terme ultime de l'Action $»^{16}$, cet Irrévélé « qui n'est pas l'Être de l'Étant, mais l'Infini » ${ }^{17}$. C'est ainsi que, dans la mesure où «source

\footnotetext{
10. Notes philosophiques diverses, p. 303.

11. Voir M. Heidegger, Sein und Zeit, Tübingen, Niemeyer, 1927, § 23.

12. Notes philosophiques diverses, p. 262.

13. Ibid., p. 263.

14. Ibid.

15. Ibid., p. 264.

16. Ibid.

17. Ibid
} 
de sens $=$ Autrui $»^{18}$ et que "Action (= orientation totale) $)$, Levinas sort décidément du caractère formellement vide du geste renversant, vide formel déjà remarqué. Il le fait lorsque le sens du virement mis en acte rétrocède en deçà, se déplace "avant", en cette orientation dont il est question quand il s'agit de sens et qui est plus direction (en ce cas déjà orientée) qu'affaire de la logique. Certes, le virement «n'enseigne rien sur l'objet de la pensée», on l'a dit, mais il est «une fonction dans la structure du sujet par laquelle le sujet est sujet séparé». Cela indique que le sujet se déplace vers ce qui le dés-oriente, c'est-à-dire l'oriente "autrement qu'être», qui lui donne un centre ne lui appartenant pas, qu'il ne possède pas, mais qui l'oriente au-delà de l'ontologie fondamentale et aussi (et déjà) «au-delà de l'essence» par une «Orientation» qui est simultanément «Action»:

$22^{\circ}$ Terme ultime de l'Action - Irrévélé. Mais qui n'est pas mystère, lequel est la négation du révélé. Le couple Révélé-Mystérieux est de l'ordre du fini - «pensée», «savoir», catégorie (opposée à l’attitude). Dans le visage qui est révélation - dimension de la hauteur.

$23^{\circ} \mathrm{La}$ dimension de la hauteur ne peut être posée comme ultime signification, bien que l'expérience de la hauteur spatiale suppose déjà la signification. L'Irrévélé - Il - qui n'est pas l'Être de l'Étant, mais l'Infini19.

Le trait déjà orienté du geste d'inversion thématique, son «n'être pas vide» se déclare ici, là où ce qui oriente l'Action - en en étant aussi son terme dernier - est cet Irrévélé qui, précédant et excédant tout horizon de manifestation, semble orienter l'Action. Dans la note $<51>$ de la liasse B des Notes philosophiques diverses, Levinas revient sur le même motif:

Le sens c'est le fait même que l'être est orienté - qu'il y a Action ou Vie. [...] Ce qui donne un sens à la vie, c'est ce qui est au-dessus de la vie. Est au-dessus de la vie, ce qui fait que la vie est entièrement orientée vers lui. [...] Être entièrement orienté, c'est agir sans chercher à être contemporain du succès de l'acte (chercher à être contemporain du succès, ce n'est pas être orienté vers). Ce vers quoi l'être est absolument orienté = l'Infini révélé dans la Hauteur, etc. ${ }^{20}$.

\section{Au-delà de l'horizon de l'être}

Il y a un lien entre l'inversion thématique et l'orientation qui enlève à cette même inversion tout caractère vide, en montrant «comment» elle
18. Ibid.
19. Ibid.
20. Ibid., p. 353 . 
jaillit d'un point qui est déjà là, qui ne se révèle ni ne se manifeste et qui, pourtant, n'exclut pas - et peut-être demande - cette perspective devançant la démarche philosophique. Autrement dit, le fait de parler d'Irrévélé en tant que point d'origine de l'orientation implique une perspective qui, elle aussi, détermine le style de la philosophie, de ses choix et de ses démarches, quoiqu'elle n'en soit pas le seul trait.

Laissant de côté la question (sans aucune prétention de scinder ce qui ne peut pas être séparé, c'est-à-dire la perspective et les choix philosophiques par lesquelles elle se traduit) et revenant à la question dont ces pages s'occupent, il serait possible de dire que le virement, revirement, inversion thématique ne dit rien de «ce qui» effectivement se passe, mais dit beaucoup du «comment cela se passe», remarquant que, si l'on change de direction à partir d'un point source de l'orientation, cette même orientation a à être conçue dans la visée que l'on envisage d'après les passages cités, donc orientation vers l'Infini, mais, aussi, en tant que orientée (pour ainsi dire) par un Infini se soustrayant à toute manifestation. Nous proposons d'entendre en ce sens un autre bouleversement décisif, celui de l'intentionnalité, puisque l'Infini est introduit en elle avec Descartes (par la reprise de l'idée de l'Infini) et avec Husserl. Et à l'encontre de Husserl, d'après lequel " [la] référence à l'infini [est] inscrite dans le noème même de la noèse ${ }^{21}$, selon sa propre thèse, Levinas remarque que:

L'infini n'est donc pas le corrélatif de l'idée de l'infini comme si elle était une intentionnalité. Dira-t-on que 'it \{l'infini\} peut affecter le Moi par le fait que le surplus insaisissable aurait une présence symbolique dans le donné adéquat? Mais la merveille du symbole ne s'explique elle-même que par le bouleversement de l'intentionnalité - par le fait que contrairement à la maîtrise parfaite de l'objet, par le sujet dans l'intentionnalité, l'Infini désarçonne son idée. Ce bouleversement consiste dans le fait que le Moi reçoit absolument, apprend absolument, non pas au sens \{socratique, une signification qu'il n'a pas prêtée, précédant toute Sinngebung ${ }^{22}$.

Dans la note que les éditeurs ajoutent à ce texte, à propos de la Sinngebung, on peut lire: "Notion husserlienne que Levinas traduit par "l'acte de prêter un sens", et dont il souligne qu'elle constitue chez Husserl la signification propre de l'intentionnalité de la conscience (cf. En découvrant l'existence avec Husserl et Heidegger, op. cit., p. 22.). La critique lévinassienne de l'intentionnalité est donc d'abord une critique de la Sinngebung ${ }^{23}$. Mais

21. Notes philosophiques diverses, p. 276.

22. Ibid., p. 277.

23. Euvres 1, p. 494, note 19. 
sur quoi peut s'appuyer une telle hypothèse de lecture? Ne suppose-t-elle pas une orientation déjà à l'œuvre? Un déjà orientant qui fait que Levinas se pose bien en deçà des gestes philosophiques accomplis, tout en ne se posant pas hors de la philosophie. Par cette orientation déjà à l'œuvre Levinas se pose «autrement» dans la philosophie, mais, toujours, «dans la» philosophie, car le fait d'être en deçà d'un geste fondateur n'implique pas le fait d'être «hors de la philosophie». L'intention de Levinas est plutôt de tenter de la penser autrement, et cela en renversant, en inversant les présupposés à partir d'un point d'orientation.

La nouveauté de cette philosophie est de s'orienter autrement que selon l'horizon de l'être, en destituant son primat. En effet, elle s'oriente à partir de l'événement dont la prophétie est faim, prophétie où l'événement "y est figuré à l'envers (наизнанку). Comme l'aliment dans la faim ${ }^{24}$. Renversement, donc, en tant que se poser «en deçà » du geste philosophique, là où l'Irrévélé oriente pour tenter l'autrement de la philosophie, jusqu'à renverser la formule socratique du «Connais-toi toi-même» en «Ignore-toi toi-même». Cependant il faudrait également se demander comment l'homme peut se savoir un sujet dé-centré, dé-localisé de soi vers un ailleurs ${ }^{25}$ ?

Le renversement en question, thématique et philosophique, inaugurant une nouvelle façon de penser, est donc aussi une question d'orientation dont le «vers où », la direction, n'est pas décidé par le sujet. Et pourtant, vers quelle pensée de l'envers est-on dirigé? Vers quel en-soi invu ce geste, originairement installé et colloqué aussi bien que philosophique, nous conduit? Vers quel en-soi, renversé pour protéger, pour garder?

\section{L'en-soi, «envers sans endroit»; ou de l'irréversibilité de l'envers}

«Un envers sans endroit»: c'est l'affirmation de Levinas dans Autrement qu'être ou au-delà de l'essence, où le philosophe emploie un lemme qui est déjà présent dès la genèse de sa pensée quand, dans la conférence «Les Nourritures», il s'interroge sur la possibilité d'aller vers un en-soi encore invu par la philosophie. Plus encore, quand il s'interroge sur la possibilité

24. Carnets de captivité, p. 80.

25. En ce sens, les mots de la note $<38>$ de la liasse A des Notes philosophiques diverses (p. 278) sont chargés d'un grand poids: «Dans les бьіјиньі,тяга земная. Or on interprète les бьіјиньі par des notions infiniment plus pauvres: nomadisme, passage du nomadisme à la vie sédentaire. Les бьіјиньi retrouvent le sens métaphysique de ces notions que l'on croit à tort ultimes. Elles touchent le fond et ne sont pas les figures des phénomènes sociaux qui seraient ultimes». 
de penser ce qui est destiné à rester le côté envers et invu, envers car il est "refusé à la vision » ${ }^{26}$. Dans Autrement qu'être ou au-delà de l'essence, cet envers est découvert par la «mise à l'envers » du sujet, plus précisément par son recroquevillement, car "son “envers l'autre" est cette mise à l'envers même ${ }^{27}$. Plus loin, Levinas nomme la douleur l' «envers de la peau», la "nudité plus nue que tout dépouillement», et l'expression revient aussi quand, dans le même ouvrage, l'hypostase, s'exposant comme soi-même à l'accusatif, offre sa propre passivité «comme un envers sans endroit ${ }^{28}$. Or, cet «envers sans endroit» semblerait être une sorte d'ab-solutus, délié et dénoué de tout rapport avec le côté endroit et visible. Un envers que l'on pourrait définir irréversible (et en ce sens "sans endroit»). La genèse de l'ouvrage rend raison de la manière dont Levinas atteint ce point si radical et cette formulation drastique, non seulement en donnant à voir le commencement de cette pensée, mais encore en montrant la mise en acte d'un geste, de cette posture renversante de la philosophie décrite jusqu'à maintenant. Cette posture sera interrogée en suivant une question directrice : l'irréversibilité de cet envers, de cet en-soi atteint par la mise en acte du renversement qu'il provoque, appartient-elle encore à l'horizon de la «manifestativité» et donc de la phénoménologie au moins historique? Autrement dit, cet en-soi qui appartient, certes, à l'ordre de la philosophie, et qui appartient aussi à l'ordre de la manifestation, relève-t-il encore de la phénoménologie?

Dans les Notes philosophiques diverses, il y a au moins trois lieux très significatifs où Levinas parle d'envers. Il introduit le terme dans la note $<2>$ de la liasse A dont le titre est Le «pour soi» du besoin:

[Le] Pour-soi du besoin, n'est pas représentation, n'est pas conscience [...], n'est pas non plus: «être en vue de sa propre existence» de Heidegger. [...] Le "pour soi» du besoin est donc un "pour soi» au sens rigoureusement égoïste où l'on dit «chacun pour soi». La jouissance est ma jouissance et exclusivement mienne. [...] Le moi du besoin, ce n'est pas «moi et pas les autres» ni l'exclusive du « quant à moi » ${ }^{29}$.

Ce moi est seul, mais «sans solitude ${ }^{30}$, car sa jouissance est «en-deçà » de toute négation. Or, c'est justement cette solitude irréversible et première qui conduit Levinas à poser la question:

26. "Les Nourritures", in Euvres 2, p. 164

27. Autrement qu'être ou au-delà de l'essence, La Haye, Martinus Nijhoff, 1978, p. 63 et 64 .

28. Ibid., p. 134.

29. Notes philosophiques diverses, p. 243.

30. Ibid. 
Comment le pour soi de la jouissance peut ne pas être relation - fût-elle négative - avec ce qui n'est pas l'être besogneux? Comment une solitude peut-elle ne pas être sur le fond d'une coexistence? Comment trouver un pour soi premier qui ne soit pas un mode déficient ${ }^{31}$ ?

La condition de cette non-relativité est l'immanence,

le fait de rester ici qui est l'événement même du pour soi, condition de la jouissance. Philosophie transcendantale au sens fort: la terre est la condition par excellence ${ }^{32}$.

Il s'agit de l'ici de l'immanence qui s'oppose au $D a$ transcendant décrit par Heidegger ${ }^{33}$.

Fait nu du pour soi, référence à Soi dans cet abandon à la base et cette passivité (non-acte). Ici central - le monde y aboutit pour s'offrir à la jouissance. Le Moi - pas relation avec l'Être, mais frisson égoïste. Ce frisson est irréductible à la négation du monde qui suppose affirmation, visée du monde, connaissance, etc. Mouvement égoïste irréductible à la négation [...] demeurer en soi en n'empruntant au monde qu'une place pour être en soi, pour être pas « en vue de» une "place au soleil», habitation, désencombrement total, s'abandonner sans penser un objet ni faire un projet, sans pouvoir. Condition de toute pensée, de tout projet ${ }^{34}$.

C'est juste après avoir introduit cette «jouissance sans savoir», "strictement subjective», par laquelle on perce déjà au-delà de Heidegger, que le cur lévinassien du renversement s'annonce, et cela à propos du savoir: «Tout savoir nous introduit dans l'intersubjectivité. Enseignement, voix de l'autre rive, l'envers du monde limité à la jouissance. S'occuper de cet envers du monde, c'est cela la raison " ${ }^{35}$. Envers en tant qu'en-soi invu "vers où » la philosophie se tente. Certes, ces quelques lignes ne suffisent pas à dire «vers où » mène le renversement de la philosophie, cependant c'est en elles que s'annoncent des motifs que d'autres conférences développeront.

Le deuxième passage où le mot « envers » apparaît est dans la note <67> de la liasse B. Ici les termes en jeu sont plus «classiques», caractérisant en propre la philosophie de Levinas. Soulignons d'abord le titre de la note:

31. Ibid., p. 244.

32. Ibid., p. 244-245.

33. «Heidegger n'a pas connu l'idée de jouissance, son pour soi. H. a identifié vérité = dévoilement = événement dans l'être» (ibid., p. 245).

34. Ibid.

35. Ibid. 
" $\underline{\text { Visage }}$ - $\underline{\text { nudité }}$ - expression - profondeur - la fin du dévoilement . Cette note 67 est publiée parmi celles dans lesquelles les thèmes lévinassiens les plus connus font leur apparition : l'infini, le rapport éthique en tant que seule possibilité de la métaphysique, la résistance éthique du visage et l'injonction «Tu ne tueras point». Injonction s'opposant à un désir d'assassiner qui n'est que « l'incompréhension du compréhensible, l'irrationalité du rationnel » ${ }^{36}$, où «rationalité» signifie:

Non pas le fait d'être saisissable. Nouveau sens de sensé et de justifié: qui n'est pas le sens d'un être, mais d'une créature. La rationalité avant moi $\{$ Loi - Rationalité de la loi plus que celle du logos\}. Une rationalité antérieure à la compréhension de l'être et au dévoilement et à la vérité. Cette antériorité à l'homme en constitue tout le contenu. Un passé absolu. Un monde comme créature. Le monde au lieu d'être Geworfenheit est raison - et raison non pas contre le passé, mais à cause du passé. C’est le passé absolu comme tel - qui est sa justification. Dans ce sens - la raison est enseignement. Elle nous vient du passé, mais d'un passé absolument antérieur à moi qui n'est pas réminiscence - mais enseignement. Le fait de m’être antérieur [...] d'un passé avant mon passé et par conséquent d'un maître - est l'essentiel de la raison ${ }^{37}$.

\section{L'infini dénudé}

Il se produit, de nouveau, un renversement thématique qui ne vise aucune irrationalité, mais qui «garde» la raison en en montrant le nouveau sens, à savoir une raison et une rationalité non centrées sur l'ego et dont le sens est l'enseignement. Cette sorte de bouleversement, ou inversion, ou renversement thématique et philosophique de la raison, introduit à une modalité "autre» de connaissance, où celle-ci est en deçà de toute possible saisie formelle et même de toute forme. Il s'agit de la connaissance inaugurée par la caresse ouvrant la possibilité même de la nudité du visage, qui est une nudité non obscène, dans la mesure où elle est une «étreinte», un «se saisir» qui est, en réalité, "le dépouillement de tout ce qui reste comme forme dans la nudité. Étreindre c'est dénuder", écrit Levinas dans un passage où on lit aussi :

[...] le visage qui se dénude dans la caresse - plus nu que la nudité n'est plus obscène et comme la forme de l'informe. Mais par là, il n'est plus ce qui sculpte un fond - mais l'ouverture elle-même comme une nouvelle dimension ${ }^{38}$.

36. Notes philosophiques diverses, p. 368.

37. Ibid.

38. Ibid., p. 370 . 
À l'encontre d'un connaître, qui n'est autre que le «surprendre quelque chose sur un fond, parcourir l'objet comme sur un fond sculpté» ${ }^{39}$, la caresse, qui ne peut que venir d'ailleurs et d'autrui, achève ce qui est non accomplissable dans la pensée et par la connaissance; elle parvient là où l'ontologie fondamentale n'aboutirait jamais, vers un fond qui est non-fond, nudité totale dépourvue de toute forme. Il s'agit de la sortie de tout horizon et de tout fond vers un non-fond qui n'est que dévêtement (et non dévoilement de quelque "chose» qui est déjà là) aboutissant au visage, c'est-à-dire «la possibilité du dévêtement total - la forme qui se démasque " ${ }^{40}$, où la nudité n'est pas le "revêtement de l'informe par une forme ${ }^{41}$, par le truchement de quelque chose qui lui préexiste, car la caresse est «le dévêtement même de la forme [dont] l'aboutissement est le visage $»^{42}$.

Le dévêtement vise un non-fond insaisissable sous tout horizon, autrement dit une radicalité irrévélable, si on la conçoit comme ce qui a à venir à la manifestation, dont Levinas parle en ces termes: «l'envers de la forme - et l'envers ne vaut pas l'endroit. [...] le visage est comme ce qui perce - c'est l'intérieur entièrement dégagé de sa forme " ${ }^{43}$. Il n'y a rien de formel qui précède sa manifestation, ni même aucune forme; le visage est - nous le répétons - cette "possibilité du dévêtement total - la forme qui se démasque " ${ }^{4}$. Aucun dévoilement n'aurait pu ouvrir sur ce point radical se passant de toute forme, mais aussi, on l'ajoute maintenant, de toute révélation. Il s'agit de l'«envers sans endroit», d'un intérieur qu'aucune forme n'exprime et qui ne se saisit pas sous un quelconque horizon, "point dernier» où l'envers met en acte lui-même le renversement, philosophique et thématique, de toute intention:

$[\ldots]$ si ce «se dégager entièrement de sa forme» - n'est pas un revêtement de forme - c'est qu'il doit être décrit comme ce qui nous vise - c'est le viser même de l'informe qui s'accomplit dans la face = le visage.

Dégagé de la forme - le visage est dégagé des limites - le visage n'est pas objet défini - mais l'infini.

Dégagé de la forme, dévêtu de toute forme, eequi app l'infini dénudé - n'est pas révélé, n'est pas dévoilé. Le dévoilement - est l'apparition d'une forme sur un fond, à l'horizon qui la délimite. L'infini est exprimé. Le visage est expression. Ce que nous avons dit sur la particularité de la nudité - revient

39. Ibid., p. 369 .

40. Ibid., p. 371.

41. Ibid.

42. Ibid.

43. Ibid.

44. Ibid. 
ici à dire qu'expression n'est pas signe extérieur, symbolisant un intérieur. Mais rapport irréductible: ni signe, ni symbole ${ }^{45}$.

Or, cet envers in-forme, ce non-fond vers lequel le renversement thématique perce, renversement que, réciproquement, il met en acte en se déclarant comme l'invisable s'annonçant en nous visant, et ne s'annonçant qu'en nous visant, appartient-il encore à l'ordre de la phénoménologie, au moins de la phénoménologie historique? Cet envers in-forme ne perce-t-il pas, ou tout au moins ne tente-t-il pas de percer, vers un autrement que la révélation et que l'apparition où se trouve conduit Levinas après avoir mûri sa pensée "en découvrant l'existence avec Husserl et Heidegger » ${ }^{46}$ ? L'effacement même des mots "ee qui app» invite, peut-être, à accomplir un pas au-delà de la phénoménologie historique, signalant que l'apparaître ne détermine pas l'ordre sur lequel cette philosophie et l'envers qu'on tente d'élucider veulent s'attester. Même l'indication que l'infini dénudé «n'est pas révélé» perce dans cette direction en déclarant qu'ici il ne s'agit plus de révélation.

"J'en viens à renverser l'ordre» ${ }^{47}$. Nous avons déjà cité ce passage où nous lisons aussi que la conscience a sa source dans l'extériorité et non dans l'intériorité. Voici un renversement qui, un pas après l'autre, conduit "hors des» catégories aristotéliciennes de l'être que la philosophie a transférées dans le moi, c'est-à-dire la passivité et l'activité. Dès lors, il y a un autre renversement dans lequel le moi lui-même se trouve renversé: «Recevoir et donner - caractéristiques ontologiques du Moi, en dehors de la passivité et de l'activité. Importance de la Matière » ${ }^{8}$. En effet, la matérialité est «l'envers de l'avoir, condition du donner - Condition du je» 49 . Le troisième passage où revient explicitement l'envers est, donc, celui-ci, où l'envers de l'avoir est condition du moi; condition, certes, particulière, car elle le dépossède de soi. Bref, il s'agit d'une condition en tant que dé-possession. La condition du moi n'est pas son «auto-possession» ou "pouvoir de» mais une matérialité - elle aussi sans «objet» et tout simplement condition de. Il semblerait que la question soit: comment le moi (n') est (pas)? Moi dont le caractère ontologique se soustrait à la formalité ontologique grecque, moderne ou à l'ontologie fondamentale. Un trait ontologique qui ne se borne qu’à dire la condition; qui rigoureusement dit «ri-en (non-ens)» et

45. Notes philosophiques diverses, p. 371-372.

46. Nous pensons ici à l'ouvrage En découvrant l'existence avec Husserl et Heidegger dans lequel Levinas déploie sa lecture originale de la phénoménologie.

47. Notes philosophiques diverses, p. 396.

48. Ibid., p. 437.

49. Ibid., p. 438 . 
le dit tout en disant «les conditions de». L'envers est ainsi cette possibilité qui précède tout autre «se donner» effectif.

Dès lors, le renversement thématique et le renversement philosophique se rejoignent dans cette thèse selon laquelle la matérialité est la condition du moi; condition qui n'est pas "quelque chose», mais la verbalité du recevoir et donner, la verbalité d'ascendance heideggérienne. Car en plusieurs lieux (que je limiterais à la citation de la partie d'Éthique et infini consacrée à Heidegger) Levinas dit que le philosophe allemand a réveillé « dans le mot être $[. .$.$] sa verbalité, ce qui en lui est événement, le "se passer" de l'être { }^{50}$. Or dans les Inédits, Levinas ne semble pas faire quelque chose d'autre, en reconduisant le moi à son envers, à cette matérialité qui est le contraire de l'avoir aussi bien que de la pure formalité; cette matérialité qui est dans les verbes recevoir-donner, matérialité qui est la condition même du donner du moi et, donc, dé-possession du moi de lui-même pour se recevoir en tant que moi à l'accusatif, selon un motif sur lequel la philosophie française a beaucoup réfléchi en ouvrant des voies différentes par rapport à celle suivie par Levinas ${ }^{51}$. Dès les Inédits s'opère donc un renversement du sujet du nominatif à l'accusatif, renversement atteignant cette matérialité qui est l'envers, de la possession et l'avoir et qui est aussi la verbalité du recevoir et du donner, condition du moi. Autrement dit, le moi n'a pas à être, car il «se passe» (tout comme l'être heideggérien, selon Levinas) par le recevoir et le donner qui en sont l'envers invu, à l'encontre de la luminescence comprenant (et comprise) de l'ego transcendantal. Le geste philosophique de Levinas, donc, dès la genèse de sa pensée, a renversé cet ego en son envers, tout en tentant de s'approcher de son (non)être, le dé-possédant de soi par la verbalité qui le donne à soi en son se recevoir.

\section{L'envers de l'horizon}

Non-fond, dé-possession, la genèse du renversement perce, peut-être, au-delà de la phénoménologie "historique». N'est-ce pas en faisant signe aussi vers un mysticisme philosophique ${ }^{52}$ ? Il y a, certes, ce risque, peut-être

50. Éthique et infini. Dialogues avec Philippe Nemo, Paris, Fayard, 1982, p. 28.

51. Parmi ces réflexions - au-delà des motifs propres à Emmanuel Levinas - il faut citer l'interloqué de Jean-Luc Marion. Voir Réduction et donation, Paris, PUF, 1989, p. 298 sq.: "Ainsi je m'éprouve - ou: je s'éprouve - comme revendiqué, assigné, convoqué à l'accusatif, privé de son droit au nominatif dénommant toute chose à la manière d'un accusé».

52. Mysticisme que Levinas se propose d'éviter, mais qui semble revenir dans le ton apophatique de son langage. Sur le refus lévinassien de la mystique, voir «Les Enseignements », in Euvres 2, p. 187: «Si la philosophie est le savoir par excellence - le savoir de la condition - il ne faut pas que l'échec de ses argumentations nous pousse vers l'irrationalisme qui se contredit en s'énonçant ou vers le mysticisme bavard de l'ineffable». 
en tant que «beau risque», à assumer pour aller vers ce que la pensée déjà à l'œuvre n'a pas encore atteint et dit en s'attestant à partir d'horizons censés être les seuls capables de rendre possible toute manifestation et en n'interrogeant que la manifestation. Cela ne semble pas être le souci principal de Levinas, comme on a pu le montrer en décrivant le dévêtement provoqué par la caresse; dévêtement différent par rapport au dévoilement qui demande toujours un fond d'où l'objet se découpe. Dans la conférence «Les Nourritures", ce thème revient instamment, notamment dans les pages centrales où Levinas parle du rôle de l'horizon dans la manifestation:

L'horizon sert certainement de fond à l'objet qui apparaît, mais l'horizon n'est pas une trouée - il est fini et fermé. L'achèvement consiste en ce que l'envers de l'horizon n'importe pas. Il impose le désintéressement à l'égard de l'au-delà. Alors que la notion cartésienne du fini se réfère déjà à l'infini, l'achèvement désigne la suffisance foncière de l'horizon. [...] Cette indifférence de l'horizon à l'égard de son envers, ce caractère en quelque manière centripète de son apparition fait que le monde tout en étant en soi est pour moi ${ }^{53}$.

Penser à l'envers implique que le renversement advient, «se passe», et ce qui est renversé thématiquement, dans cette conférence, c’est le point de vue de Heidegger, d'après lequel la compréhension reste un pouvoir congruent avec le primat moderne de la liberté, l'un et l'autre étant liés à la prééminence de la vision et ignorant que «le monde s'offrant à la vision est nourriture " ${ }^{54}$. Ces nourritures qui "représentent un plan d'être distinct et de l'instinct et de la raison - où la conscience est simplement la gratuité de cette jouissance, sa clarté, sa vision ${ }^{55}$. L'analyse de la lumière dénonce ici le primat de l'immanence et de la clôture qu'elle détermine: "La lumière n'est pas l'élément de la transcendance, même au sens heideggérien de ce terme. Elle décrit une immanence. Ce qui est illuminé est achevé et défini ${ }^{56}$. Et c'est justement l'horizon, c'est-à-dire ce qui est achevé et définit, notamment l'horizon de la conscience, que l'indifférence à l'égard de l'envers, par l'oubli des prolongements et des revirements, oppose - par sa certitude lumineuse, certitude de son propre savoir aussi bien que de soi - à l'inconscient. En effet, c'est la conscience qui «coupe, en quelque manière, les rapports entre ce qui est révélé et son envers ${ }^{57}$ : tandis que «le

53. «Les Nourritures», p. 158-159. Tandis que - disons-nous, dans la démarche de Levinas l'en-soi cherché par notre philosophe fait que je découvre que "moi, je suis pour et par", et cela inverse la question.

54. Ibid., p. 156.

55. Ibid.

56. Ibid., p. 158 .

57. Ibid., p. 160. 
monde est donné, c'est-à-dire \{est\} sensible», il «nous échappe dès que la pensée s'y applique», car la lumière «lui assure cet achèvement qui arrête les questions ${ }{ }^{8}$. Là où manque l'envers il n'y $\mathrm{a}$, donc, qu'achèvement, clôture, fermeture, arrêt - bref, le contraire de toute ouverture, et même la sensibilité, "à l'aide de cette suffisance ou de cet achèvement de la lumière» se définit improprement, voire "indépendamment de toute référence aux organes des sens et en dehors de toute notion de réceptivité " 59 . Autrement dit, la sensibilité - de part à part réceptive - en vient à être conçue en dehors de la réceptivité qui lui appartient, ce qui ne fait d'elle qu'une «conscience, mais foncièrement distincte de la raison ${ }^{6}{ }^{6}$. Or sortir du sensible conçu est possible si «l'on s'élève aux formes catégoriales » pour penser l'envers, car «dès que l'on s'élève aux formes catégoriales on ne peut se contenter de l'endroit sans rechercher l'envers ${ }^{61}$.

Il se pourrait qu'ici Levinas vise même l'élargissement de la sensibilité, ouverte intérieurement en son invu ne pouvant se manifester dans le cadre ébauché par n'importe quel horizon. Cet invu qui est l'en-soi - envers dont on tente l'approche et toujours à penser - est pourtant irréversible, ouverture dernière qui ne s'annonce que par la sortie de l'horizon conscientiel. Une nouvelle fois, à l'encontre d'un pouvoir et d'une liberté reposant sur le primat de la vision, le catégorial «se situe au-delà » de la vision elle-même. Et pourtant, au-delà aussi de la soi-disant opposition traditionnelle entre activité et passivité, pensée toujours more aristotelico en tant qu'agir et subir. Dès lors, la réécriture par Levinas - au-delà des catégories grecques - se précise par cette remarque signalant que le catégorial ne se caractérise pas "par l' "activité" du sujet, alors que le sensible se caractériserait par la passivité». Tout au contraire, "c'est la sortie du plan "se suffisant" du subjectif qu'atteste l'intellectuel. Il atteste une altérité»" ${ }^{62}$. On arrive alors à l'affirmation dans laquelle ce qui est véritablement en jeu dans la philosophie de Levinas s'annonce par la reprise du motif de la lumière qui "décrit l'horizon sans envers où se place toute prise de conscience. Cette absence d'envers distingue la sensibilité de la pensée. La sensibilité n'est pas pensée, mais jouissance ${ }^{63}$. Renverser cette luminiscence vers l'invu force la philosophie à aller vers une pensée de l'activité et de la passivité différente en sa racine par rapport à celle déjà pratiquée, visant aussi la redéfinition de la sensibilité et de la pensée, ne relisant pas un mouvement

58. Ibid.

59. Ibid.

60. Ibid., p. 161.

61. Ibid.

62. Ibid.

63. Ibid. 
de «revirement ${ }^{64}$ renversant une vision et une jouissance indifférentes par rapport à la vérité "en raison même de l'achèvement de leur horizon " ${ }^{65}$, et donc indifférentes à la vérité lorsqu'une pensée de l'envers manque.

\section{La liberté nue}

La pensée manque la vérité là où elle manque l’altérité: cela est explicitement annoncé par Levinas lorsqu'il parle de la raison dans la note $<2>$ de la liasse A des Notes philosophiques diverses. Levinas veut envisager une raison qui n'est pas le pouvoir suprême de l'homme et qui se situe "dans une relation qui n'est pas pouvoir», renversant donc le sens de la compréhension heideggérienne, aussi bien que le primat de la vision, fondé sur le pouvoir et la liberté. Cette raison selon Levinas «suppose le monde intersubjectif de l'enseignement où une voix "venant de l'autre rive" nous annonce l' "envers" des choses, essentiellement refusé à la vision " ${ }^{66}$.

Penser à l'envers pour penser l'envers revient donc à restituer à la raison la possibilité de penser, non d'une façon centripète et autoréférentielle, mais d'une façon centrifuge, ouverte à l'autre. Il s'agit donc d'une raison effectivement élargie par et à l'intersubjectivité et qui ne se réduit pas à la découverte de l'alter ego, puisque le geste de renversement de la philosophie est un geste qui trouve et gagne un monde intersubjectif et non egocentré. Voilà, donc, que l'enseignement revêt un rôle central dépassant la vision; cet enseignement d'où procède «le mouvement de la pensée qui aperçoit le fini sur un fond infini $"{ }^{67}$. Penser à l'envers revient ici à procéder d'un autre ordre, à penser «contre toute la philosophie contemporaine ${ }^{68}$, à penser pour chercher l'" autre", "vers où " la philosophie de Levinas conduit. Penser à l'envers signifie "être mis en condition de penser", et c'est ce que nous apprend la conférence "Les Enseignements", dans laquelle Levinas s'interroge sur le moment où vie et réflexion s'ouvrent l'une à l'autre dans cette situation qu'il appelle "enseignement» ${ }^{69}$. Par l'enseignement, le mouvement de la venue de l'autre et l'ouverture de l'horizon coïncident avec la critique d'une temporalité de la jouissance et de la lumière, temporalité sans envers ne privilégiant que le présent. On ne parle pas, ici, d'achèvement de l'horizon sans envers, mais il y a autre chose, et peut-être plus, c'est-à-dire la critique de l'auto-possession intégrale

\footnotetext{
64. «Les Nourritures", p. 159.

65. Ibid., p. 164.

66. Ibid.

67. Ibid., p. 158-159.

68. "Les Enseignements», p. 178

69. Ibid.
} 
du moi pour lequel, à partir d'un passé qui n'est reconnu qu'en fonction du présent ${ }^{70}$, «Autrui - ne [le] concerne pas. Il est l'étranger. [...] Autrui m'est étranger ${ }^{71}$. Par cette auto-suffisance, le moi se découvre à partir de soi et non à partir d'autrui, situation que Levinas inverse afin d'invertir le paralogisme inauguré par le pouvoir moderne de la liberté: «dans le désir de soumettre à notre liberté notre existence même, réside [...] un paralogisme. La liberté et le pouvoir sont dirigés sur le monde - ils sont l'action; les exiger pour les conditions mêmes de l'action, c'est leur prêter un emploi qui n'est pas le leur $"{ }^{72}$. À l'encontre de ce contre-raisonnement, de ce raisonnement fallacieux, «l'inversion première de la réflexion» que Levinas propose est non seulement un renversement thématique effectué afin de penser différemment cette situation, mais il s'agit aussi d'une inversion en tant que pénétration en deçà (tout comme en deçà était l'envers ignoré par l'horizon lumineux). Il s'agit donc encore une fois d'un penser à l'envers vers un envers invu, mais par lequel «le drame de n'avoir pas choisi son existence se dénoue » ${ }^{73}$. Tandis que la liberté des "philosophes contemporains", affirmant son propre primat, «sous le regard d'autrui » craint de «se laisser traiter comme chose dépourvue de liberté» ${ }^{74}$, le regard que l'autre fait affleurer coïncide avec cette nudité (dévêtement radical mis en acte par la caresse) qui est l'envers d'une liberté lumineuse. Voilà un envers que l'inquiétude que j'éprouve face au regard d'autrui porte à la lumière par un parcours où je découvre que mon droit même à la liberté

m’apparaît comme contestable, [...] comme si l'être dont dispose le sujet libre n'était pas à lui, comme si le monde où il se trouve n'était pas sa patrie, mais un exil, comme si ce monde était toujours aux autres et comme s'il fallait me le promettre et me le donner, comme si ma liberté était totalement nue ${ }^{75}$.

Ce moi souverain, déjà envisagé en sa verbalité, se découvre maintenant créé et élu: «Non pas que le moi existe d'abord et soit élu ensuite - son ipséité réside dans son élection. Son élection est sa création ${ }^{76}$, l'une et l'autre à investir originairement la liberté, passé absolu dont aucune réminiscence ou souvenir n'est possible, vers lequel il n'y a que l'enseignement qui y perce, en brisant l'auto-certitude et le pouvoir de toute auto-compréhension:

70. Voir ibid.

71. Ibid., p. 179.

72. Ibid., p. 184 .

73. Ibid.

74. Ibid.

75. Ibid.

76. Ibid., p. 185 . 
«J'appelle la façon dont ce passé absolu de mon élection et de ma création peut m'être donné - enseignement ${ }^{77}$.

Si la conférence «Les Nourritures» questionne et renverse la raison, dans «Les Enseignements», Levinas questionne et renverse le savoir, celui-ci étant renversé par cette inversion première de la réflexion qui n'est autre que l'enseignement, «cette pénétration en deçà de sa condition pour justifier sa condition est dans sa structure la plus formelle - le fait du savoir ${ }^{78}$. Cette pénétration est un «se tourner vers sa propre condition » 79 , un mouvement, une inversion autre aussi bien par rapport au jugement que par rapport au dévoilement. Et la vérité du savoir, d'après cette inversion qui est pénétration, est «inadéquation [...] de la réponse et de la question ${ }^{80}$, vérité $\mathrm{du}$ savoir et savoir lui-même qui, toutefois, ne peuvent pas être assumés, car la question posée par l'élève, chez qui la réponse du maître est accueillie, est toujours une autre et nouvelle question ${ }^{81}$. Il s'agit là de changer les notions traditionnelles de la philosophie, notamment celle de l'être et de l'être libre, incompatible avec l'idée de transcendance relevant de la création ${ }^{82}$. Une transcendance que Levinas situe «en dehors de l'événement de l'être» et qui est relation ${ }^{83}$, relation avec l'autre ${ }^{84}$, relation érotique par laquelle l'exister du moi est une existence pluraliste, car « dans la relation érotique, le sujet ne se saisit pas à partir de lui-même» ${ }^{85}$. Plus loin: "Dans l'exister lui-même, il y a une multiplicité et une transcendance ${ }^{86}$, jusqu'à une fraternité d'où jaillit une nouvelle idée de collectivité. Par rapport aux lignes initiales de la conférence, la situation se renverse: en effet, l'inversion en pénétration opérée par Levinas rend possible l'idée que le moi se découvre en tant que moi qui n'est pas autarcique ou auto-suffisant, moi de la relation qu'Autrui vise "en tant qu'il entre dans [son] monde ${ }^{87}$. Il ne s'agit pas seulement du changement du point de départ, mais de l'invention, dans le sens étymologique du mot latin invenire, de l'envers du moi, envers qui est la relation découverte par un mouvement dont l'enjeu sont la raison et le savoir conçus selon de nouvelles figures, autrement dit, à l'envers: il ne s'agit

77. «Les Enseignements», p. 185 .

78. Ibid.

79. Ibid., p. 186.

80. Ibid., p. 186-187.

81. Voir ibid., p. 187.

82. Ibid.

83. Ibid., p. 189.

84. Ibid., p. 190.

85. Ibid., p. 192.

86. Ibid.

87. Ibid., p. 179. 
pas du moi achevé et auto-suffisant, mais du moi créé, élu, originairement investi par un passé absolu.

Mais qu'est-ce qui peut mettre ces affirmations à l'abri d'un glissement vers un certain mysticisme, même si Levinas déclare explicitement vouloir l'éviter ${ }^{88}$ ? Ce passé absolu, dénonçant l'inadéquation de tout horizon, doit en inaugurer un nouveau et propre à lui pour venir à la manifestation, ou bien ne sort-il pas de la possibilité même de se phénoménaliser? Nous nous bornons à cette remarque, qui est une sorte d'anticipation de ce trait absolu que les Inédits ont déjà annoncé et qui caractérisera successivement l'envers (sans endroit) d'Autrement qu'être ou au-delà de l'essence, signalant que cette pensée, renversant la philosophie, pose au moins le problème de la «phénoménalisation» d'un envers pensé si radicalement et irréversiblement. Un envers sans endroit risque en effet l'impossibilité de se penser autrement qu'éthiquement.

\section{Parole et existence}

C’est aussi à propos de l'intentionnalité que Levinas souligne la nécessité d'invertir le mouvement que la philosophie a pensé a contrario, car sa thèse est que le mouvement de celle-ci est «l'inverse de la relation avec le dehors » ${ }^{89}$. Contrairement à Sartre, pour lequel la portée "fondamentale» de l'intentionnalité est dans le fait qu'elle rend possible l'éclatement de la conscience vers l'extérieur ${ }^{\circ}$, décidant de la fin de la philosophie de l'immanence de la conscience, pour Levinas l'intentionnalité marque le mouvement opposé, contraire, car elle «tisse mon intériorité»:

L'intentionnalité qui anime la perception délimite et dessine mon habitation dans le monde. Comme la maison que j'ai construite, mais que j'habite en me fermant d'abord sur l'extérieur, le monde décrit ou construit par l'intentionnalité, meublé de mes produits, m'enferme dans mon horizon. [...] Habiter, c'est se fermer sur l'extériorité, vivre dans un horizon limité dont l'au-delà est oublié, ne pas se poser devant le fini la question de l'infini, rester dans

88. On peut comprendre la remarque de Levinas et son opposition au mysticisme de l'ineffable (voir supra, note 52), mais il reste toujours difficile de se soustraire et de le soustraire au beau risque de ce mysticisme-là où le langage négatif a à se formuler positivement dans le renversement des termes classiques de la philosophie par le recours à des mots dont la «forme» est négative, qui laisse entrevoir par la désorientation le retour du mysticisme.

89. «L'Écrit et l'Oral», in CEuvres 2, p. 206.

90. Voir J.-P. Sartre, Une idée fondamentale de la phénoménologie de Husserl: l'intentionnalité, in Situations, I, Paris, Gallimard, 1947, p. 31-35 (l'article remonte à 1939). Voir également La transcendance de l'ego, Paris, Vrin, 1936 et L'imagination, Paris, PUF, 1936. 
l'immédiat sans rechercher la médiation: [...] une maison, ce n'est pas s'en servir seulement ${ }^{91}$.

L'achèvement de l'horizon, son accomplissement ignore l'au-delà, renferme sans ouvrir ni percer. Cependant, il se passe ici aussi quelque chose d'autre. Si la philosophie a à être renversée pour saisir la question de l'infini au-delà du fini, ce geste a à être mis en acte, à cause du fait que le phénomène - même dans l'annonce qu'en fait le $\$ 7$ de Sein und Zeit - demeure absent en son apparition. Autrement dit, le phénomène, dans les figures que la philosophie en a formulé, n'apparaît pas, reste absent et manquant. Or il faut à Levinas un sol pour pouvoir dire cela, et ce sol c'est l'action humaine, à propos de laquelle il dit qu'elle « exprime l'homme [...] Mais cette expression est à peine une expression. Il est évident que l'intention d'exprimer - ce rapport unique avec l'extériorité, manque à l'expression spontanée de l'action ${ }^{92}$. Ainsi en toute œuvre "on devine» l'intention qui l'a animée, mais par elle nous ne sommes que (voire: notre intention est) jugés "par contumace", tandis que moi, l'auteur, je reste absent:

On a certes deviné dans mon œuvre mon intention, on a même deviné peutêtre mon intention réelle, mais on m’a jugé par contumace. J’ai été absent de mon procès, je n'ai pas pu porter secours à moi-même: je n'ai pas parlé. On a pénétré dans mon intérieur en mon absence ${ }^{93}$.

Ce qui manque, ici, est la parole, et sans la parole, «on pénètre dans ma vie, mais on ne se met pas en relation avec moi; sans la parole qui me sollicite et à laquelle je réponds, je n'ai pas d'existence véritable - pleine et extérieure " ${ }^{4}$. Levinas ajoute: «S'exprimer uniquement par sa vie, par son action, par son œuvre, c'est donc rester absent de l'être " 95 , car «le souci - et le "pour soi" - de mon activité n'expriment pas, mais constituent seulement mon intimité ${ }^{96}$. À l'encontre de l'expression qui accomplit la relation avec l'Extérieur, «l'intentionnalité de l'acte, de la visée théorétique - font partie de mon intimité » ${ }^{97}$, et l'intentionnalité, on le sait, «m'enferme dans mon horizon ${ }^{98}$. Cette situation,

\footnotetext{
91. «L'Écrit et l'Oral», p. 206-207.

92. Ibid., p. 205.

93. Ibid.

94. Ibid., p. 206.

95. Ibid.

96. Ibid

97. Ibid.

98. Tout comme le dit le passage déjà cité, note 91.
} 
où je suis découvert mais où je ne suis pas exprimé, où j'apparais mais suis absent de mon apparition - décrit assez exactement le sens du phénomène: le phénomène est un être qui apparaît mais demeure absent. Le phénomène n'est pas une apparence - il est la réalité, mais c'est une réalité qui manque encore de réalité 99 .

Penser à l'envers afin d'atteindre l'envers revient dès lors à penser la réalité en sa véritable présence; en sa présence qui reste absente là où elle n'est pensée qu'à partir d'un horizon achevé et, donc sans envers. Une véritable présence, ou vérité, qui - d'après la leçon de la conférence «L'Écrit et l'Oral» - ne se manifeste qu'oralement "à celui qui peut poser des questions » ${ }^{100}$, prolongeant ainsi la conférence consacrée à l'enseignement. Dès le début de la conférence, Levinas remarque qu'écrit et oral sont « deux façons pour la vérité de se manifester ${ }^{101}$, et, juste après, il affirme le primat de la parole par rapport à l'écriture qui ne donne qu'une vérité relative, périmée ${ }^{102}$.

L'envers de cette relativité du vrai, qui n'est rien d'autre que la "présence de la pensée elle-même, en dehors de toute relativité ${ }^{103}$, est "sa présence dans la parole du maître, car lorsque le maître parle, la pensée a un visage ${ }^{104}$. Plus loin, Levinas écrit que le visage est la modalité de manifestation de la parole. Parmi les passages consacrés à la question du visage, nous nous bornerons à en citer un, célèbre, où se montre à nouveau le renversement de la philosophie que notre philosophe met en acte par ces conférences: "Le visage, c'est la possibilité pour une substance d'être

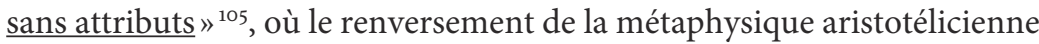
(certes, pas plotinienne) est évident. Qu'il s'agisse d'un renversement mis en acte par ce penser à l'envers évoqué jusqu'à maintenant, c'est ce que Levinas confirme par les lignes suivantes:

99. «L'Écrit et l'Oral», p. 207. Plus loin: «Que peut être cette réalité encore absente de la réalité? Ce qui reflèten elle? est caché? Mais c'est lui tonner \{prêter\} par rapport là l'eard\} du phénomène - le rapport \{<qu'a? $>\}$ \{que\} que le phénomène eonserve \{a\}à l'égard de l'apparence. [...] C'est poser la chose en soi comme un phénomène caché. Le dévoilement renvoie toujours à des phénomènes. L'expression n'est-elle pas la présence de la chose en soi qui, précisément, n'est pas sous l'apparition \{de cette chose\}?» (p. 207-208). Ce passage confirme, pourtant, notre hypothèse: le renversement, renversant l'horizon vers son envers, en-soi irréversible, n'ouvre pas ce mouvement au-delà de la phénoménalité et, donc, de toute phénoménologie au moins «historique»? Cet en-soi ne se manifeste pas, car en ce cas, il appartiendrait encore à l'ordre du dévoilement et non du dévêtement, auquel n'accède que la caresse.

100.Ibid., p. 203.

101. Ibid.

102. Voir ibid., p. 214.

103.Ibid., p. 217.

104. Ibid.

105.Ibid., p. 218. 
Le visage, c'est ce qui rend possible l'existant comme existant - comme identité - en dehors de l'horizon. Il ne s'y réfère qu'à lui-même comme un absolu. Le caractère absolu d'autrui exclut la relativité inscrite dans la connaissance où l'objet connu sort de son absolu par le fait d'être connu ${ }^{106}$.

Et autrui, remarque encore Levinas, «notons-le en passant - dans l'absolu de l'invocation est ainsi l'être inviolable du rapport moral ${ }^{107}$. Dans la conclusion de la conférence, les passages confirmant le parcours proposé (que résume bien la formule "penser à l'envers pour penser l'envers », et donc pour penser à nouveau frais la raison, le savoir, la pensée le renversant et, d'abord, renversant le moi achevé par un horizon en moi constitué par la relation) se multiplient: "La raison est un visage», "Ce qui est en relation avec nous sans être objet d'une activité pratique, sans être vision dans un horizon ${ }^{108}$ - c'est un visage. La parole est la pensée dans un visage. L'origine de la vérité est dans l'enseignement. Le visage est condition de vérité ${ }^{109}$. Cependant, c'est juste dans ces passages proposant ce renversement radical que le Leitmotiv de l'absolu revient, et peut-être pas par hasard: absolu de l'invocation - car "poser une question ne suppose pas seulement qu'on connaît autrui ou qu'on connaît sa présence, c'est aussi l'invoquer ${ }^{110}$. Tel est cet absolu du passé, passé " pas caché, car le caché est en principe dévoilable " ${ }^{111}$ (et, on le sait, la vérité qui se produit comme dévoilement est la vérité du phénomène et non la vérité du visage, à dévêtir et non à dévoiler). De ce passé, absolu, envers par rapport à un horizon qui est " endroit sans envers", il n'y a aucune phénoménalisation possible; la vérité du phénomène, vérité en tant que dévoilement, n'est pas la vérité du visage, dont la vérité est vérité de parole, d'enseignement, de relation.

\section{Le renversement est le temps}

Accomplissons-nous un pas de trop en disant que cette absoluité, cet être délié, ab-solutus, vient de la séparation, de la rupture désignant en propre la métaphysique? L’Absolu est séparé, délié, dégagé, autre. Il est en-soi ${ }^{112}$

106. «L'Écrit et l'Oral», p. 218.

107.Ibid.

108. Et donc sans envers.

109. «L'Écrit et l'Oral», p. 223.

110.Ibid., p. 217. À propos de ce trait absolu: «Le caractère absolu d'autrui exclut la relativité inscrite dans la connaissance où l'objet connu sort de son absolu par le fait d'être connu " (p. 218).

111.Ibid., p. 212.

112. Le lemme «en-soi » revient dans les pages où l'on dit que le visage, présent sans apparaître («L'Écrit et l'Oral», p. 218), «rend possible l'existant comme existant - comme identité - en 
en tant qu'envers "sans endroit», irréversible. Il ne s'agit pas de réifier l'absolu, tout comme Levinas exclut décidément que le moi dont il parle soit réifié ${ }^{113}$. Au contraire, il s'agit du fait même d'être séparé, de cette séparation sur laquelle porte la conférence du 3 décembre 1957. Même si Levinas ne le dit pas en ces termes, dans l'accent qu'il pose sur le préfixe «méta» de la métaphysique, on entend l'écho du sens adverbial du mot, indiquant en grec "entre-deux»"14. Il s'agit d'un interstice irréductible et censé créer la relation, de cette rupture minimale et irréductible faisant que l'envers n'est pas simplement «l'autre côté de l'endroit», mais ce qui contraint la pensée même aux inversions et aux renversements mis en lumière, c'est-àdire au renversement de la philosophie. Voire, il s'agit de ce qui provoque ce renversement. C'est ainsi que la non-réification de l'absolu renvoie à la non-réification de l'envers, celui-ci étant à penser plutôt comme une dé-possession invertissant qui, ce faisant, annonce, à l'encontre d'une totalité déjà pensée, l'ouverture irrémédiable à l'Autre et Autrui. Dès lors, voilà cette ouverture qui annonce en effet une séparation à l'encontre d'une totalité déjà pensée. Voilà un renversement qui renverse en même temps l'ordre ontologique de l'unité de l'être et une modalité - hégélienne - d'entendre le lien de la pensée avec l'être. Renversement qui est le temps ${ }^{115}$ :

L'intériorité qui rend possible tout cela coïncide avec le renversement de l'ordre ontologique de l'unité qui est celui de l'être sans séparation. Ce renversement est le temps ${ }^{116}$.

Ce qu'il faut souligner ici, c'est le point d'ancrage de l'argumentation qui mène vers ce renversement, c'est-à-dire la possibilité de penser la «séparation radicale», «le Transcendant de la métaphysique» qui, se posant "absolument ailleurs», "ne provient pas de la négativité» ${ }^{117}$. Une telle séparation radicale inaugure une relation non totalisée par le sujet et demeure toujours "au-delà », relation où le Transcendant, l'Autre, reste l'absolu de la relation.

dehors de l'horizon. Il ne s'y réfère qu'à lui-même comme un absolu. Le caractère absolu d'autrui exclut la relativité inscrite dans la connaissance où l'objet connu sort de son absolu par le fait d'être connu. Autrui - [...] à jamais étranger à toute emprise de la connaissance et de l'action, toujours en soi, à qui l'on ne peut que parler. Rien ne peut se superposer à cette relation d'invocation où l'en soi d'autrui est abordé» (ibid.). Ineffable, la parole qui aborde Autrui est «en dehors de toute référence à une pensée préexistante qu'elle aurait à exprimer - comme une relation originelle et fondamentale dans l'être» (p. 219).

113. Voir «La Séparation», in CEuvres 2, p. 275.

114.Ibid., p. 265.

115.Ibid., p. 269.

116.Ibid. Le «tout cela» veut indiquer la redéfinition radicale de la métaphysique.

117.Ibid., p. 267. 
[...] en se refusant à la synthèse qui réunirait en totalité le Même et l'Autre, le Transcendant, l'Autre ne supprime pas toute relation. [...] En aucune façon ils ne constituent une totalité - Et cependant ils sont en rapport. [...] Le rapport avec le transcendant est un rapport avec ce que l'être séparé ne peut contenir. La séparation est précisément cette incommensurabilité. Le rapport lui-même ne détermine en rien le Transcendant, mais au contraire confirme son extériorité. [...] L'extériorité absolue de l'être extérieur ne se perd pas purement et simplement du fait de sa manifestation. Il s'absout de la relation où il se présente, reste absolu dans cette relation ${ }^{118}$.

Un des motifs principaux de l'œuvre d'Emmanuel Levinas, à savoir la séparation et la rupture de la totalité, après s'être annoncé, se déclare et se désigne ici comme le renversement qui, on l'a dit, "est le temps ${ }^{119}$.

L'ordre chronologique - le temps - est précisément l'articulation, dans l'être même, de la séparation. Le temps est le fait que l'être n'est pas d'un seul coup, que tout, même la Cause du tout, est encore à venir et, par conséquent, conditionnée dans l'effet qui l'accueille, c'est-à-dire la connaît. La connaissance se réfère, en effet, au temps dans la mesure où en elle s'opère le renversement de l'ordre tel qu'il est en soi en ordre tel qu'il est pour la connaissance - et qui n'est pas le même. [...] La connaissance est la réversibilité de l'ordre ontologique $^{120}$.

L'enjeu est à nouveau strictement philosophique, car ce qu'on questionne est une nouvelle démarche de la connaissance. Mais ne faut-il pas souligner en même temps le trait d'absoluité qui revient instamment? Il ne s'agit pas d'un absolu réifié ou conceptualisé, mais d'un absolu en tant qu'irréductibilité absolue. Dès lors, par l'avènement de ce trait absolu de l'envers "sans endroit», nous sommes menés près de cet en-soi de la philosophie que la connaissance saisit, à nouveau, moins par une totalité assimilable et pensée, que dans la mesure où une sorte de réversibilité se donne à penser pour être renversée vers son irréversibilité. Il s'agit d'une connaissance qui connaît par l'avènement de la réversibilité même de l'ordre ontologique et non par la préexistence de l'horizon ontologique, consentant l'avènement de cet envers qui ne se laisse saisir que par la séparation. Or c'est par cet envers que «l'extériorité absolue de l'être extérieur ne se perd pas purement et simplement du fait de sa manifestation ", d'où vient que, comme on l'a déjà dit, «il s'absout de la relation où il se présente, reste

118. «La Séparation», p. 267-268.

119.Ibid., p. 269.

120.Ibid., p. 269-270. 
absolu dans cette relation ${ }^{121}$. Mais, il vaut la peine de le remarquer, il «ne se perd pas purement et simplement du fait de sa manifestation", car la manifestation n'est pas la plus appropriée à ce qu'on est en train de dire. Bref, la radicalité du préfixe "méta», de ce trait qui sépare irrémédiablement la "physique», reste-t-il encore dans l'horizon de la manifestation ou bien ne se pose-t-il pas dans cet «ailleurs» maintes fois évoqué? Et la réversibilité, ne s'inverse-t-elle pas en un irréversible non "manifestable»?

Tout comme le «méta» de la métaphysique, le «méta» irréductible précédant le phorein de la métaphore ouvre, ou peut-être met lui-même en acte, un autre renversement radical, celui-ci aussi déjà maintes fois évoqué selon d'autres biais, de l'intentionnalité, renversement qui, dans la conférence consacrée à la métaphore, est bouleversement radical vers une sorte de contre-intentionnalité. En cette conférence, très dense, où la métaphore par excellence est «l'idée d'infini » ${ }^{122}$, le mouvement jusqu'ici proposé s'enrichit d'un trait conclusif, et peut-être récapitulatif, le trait éthique. La relation, argument désormais strictement philosophique, se fait responsabilité pour l'autre, car «l'Autre provoque un mouvement éthique dans la conscience ${ }^{123}$. Plus loin, «notre analyse a essayé de montrer que la métaphore par excellence ou l'idée de l'infini est de la signifiance éthique»" ${ }^{124}$, qui, pourtant, «comporte un surcroît inadéquat à l'intentionnalité» provoquant un bouleversement radical de l'intentionnalité. Bouleversement radical, car toute signification que le Moi a apprise n'est, à vrai dire, que reçue, précédant toute possible Sinngebung ${ }^{125}$. La signification elle-même, pour sa part, n'est qu'un «renversement d'un monde dont je suis le centre en un monde dominé par Autrui ", là où la signification ne consiste pas en la Sinngebung, mais la précède, suivant le même mouvement de bouleversement dont parlait la note $<34>$ de la liasse A des Notes philosophiques diverses ${ }^{126}$.

\section{Conclusion}

Le renversement de la philosophie aboutit à ce point, invu de la philosophie, là où Autrui renverse la centralité du moi inaugurant le primat de l'éthique, et fait de ce moi constituant, un moi constitué, de ce moi qui vise, un moi visé. Découverte de la véritable intersubjectivité dans le saisir l'autre comme

121.Ibid., p. 268.

122. «La Métaphore», in Euvres 2, p. 344.

123.Ibid.

124.Ibid

125.Ibid., p. 345 .

126. Voir Notes philosophiques diverses, p. 277. 
moi ${ }^{127}$ et non dans le « saisir un autre moi (ou un alter ego) hors de moi » ${ }^{128}$, selon les descriptions de Husserl. Ainsi se découvrent des significations de la pensée, de la raison et du savoir, nouvelles, inédites, encore invues, mais, par ce mouvement propre à Levinas, destinées, peut-être, à demeurer invues. Elles sont saisies par la philosophie « renversée», mais soustraites à la manifestation. En commençant la lecture de ces pages, on affirmait que ce renversement est mis en acte afin de saisir un envers encore non saisi. Dans les conférences prononcées au Collège philosophique, Levinas parle de cet envers en le donnant à voir comme ce qu'une pensée dont l'horizon est achevé, se passant de tout au-delà, manque. Pourtant, où mène la saisie d'un tel envers? Cela mène, et Levinas le remarque, hors de la philosophie de l'existence, hors de la métaphysique de la substance et hors de toute philosophie de la totalité. Bref, là où il renverse l'ordre ${ }^{129}$, là où il renverse les termes ${ }^{130}$, Levinas est conduit vers le rapport moral, vers l'éthique, selon ce qu'un passage déjà cité annonce:

J'en viens à renverser l'ordre. Ce n'est pas ce qui est infini qui est extérieur, mais ce qui est extérieur qui est infini. L'être - c'est se rapporter à l'extérieur. Le rapport avec l'extérieur - le rapport moral - est la condition de la liberté. La communication des individus est la source de la conscience ${ }^{131}$.

Le «vers où », donc, est connu et clairement dit en ces conférences: vers l'éthique, vers la justice, vers un moi de la responsabilité, vers le primat de l'invocation et de la parole. Entre le début et la fin du parcours, entre un horizon sans envers et le point d'arrivée de l'œuvre de Levinas, il y a, pourtant, la traversée de la phénoménologie historique et la lecture de Husserl et de Heidegger. Au-delà desquels Levinas s'avance, évidemment, renversant l'intentionnalité, sortant de l'ontologie et allant au-delà de l'apparition du phénomène. Dès lors, le renversement de la philosophie coïncide-t-elle aussi avec la sortie de la phénoménologie historique? Dans les six notes publiées dans la liasse B (54-59) des Notes philosophiques diverses, Levinas définit la phénoménologie comme «description, mais une description intentionnelle», comme "accès à l'état [qui] fait partie de l'étant. Mais [...] l'étant est une des formes polarisations de l'être», comme pensée par laquelle « une idée est n'arrive pas à tépasser se séparer des voies qui y mènent ${ }^{132}$.

127. Notes philosophiques diverses, p. 477.

128. Ibid.

129. Voir ibid., p. 396.

130.Ibid., p. 411.

131.Ibid., p. 396.

132. Ibid., p. 355-356. 
S'agit-il du même mouvement qu'il inaugure par la genèse de sa pensée? S'agit-il du même geste ou bien, au contraire, ce que l'on cherche est-il justement la séparation? On n'a pas suivi le mouvement opposé afin d'aboutir à une pensée instituée, trouvant sa propre voie par un enseignement reçu. Pour aboutir à la séparation et à l'extériorité, pour en sortir de la philosophie déjà pensée, pour parler à nouveau d'un envers «oublié», pour le redonner à penser en le gardant, selon le sens de l'Umschlag, il fallait que Levinas sépare la voie et l'idée, renversant la philosophie et interrogeant la racine même de la phénoménologie, donnant à penser un envers invu afin de percer «vers où » la philosophie n'était jamais arrivée, la renversant et renversant ses horizons classiques vers de nouveaux commencements. De commencement en commencement, selon des commencements qui n'auront jamais de fin.

Carla Canullo Université de Macerata (Italie) 